\title{
How to manage fatigue in fibromyalgia: nonpharmacological options
}

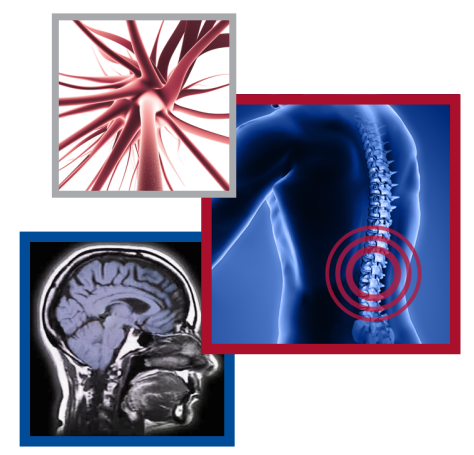

\author{
Anna Ericsson ${ }^{1,2} \&$ Kaisa Mannerkorpi ${ }^{*, 1}$
}

\section{Practice points}

- Several types of exercise have been shown to diminish fatigue in fibromyalgia (FM). For best effects, the exercise needs to be regular and performed over a longer time.

- Meditative movement therapies have shown to diminish fatigue and improve sleep in FM.

- Sleep hygiene should be included as a part of the treatment of fatigue in FM.

- Educational programs, designed to enhance self-efficacy for management of health problems is assumed to diminish symptoms in FM, including fatigue.

- Person-centered approach appears to be crucial in the treatment of fatigue in FM.

The aim of this article is to suggest recommendations for the management of fatigue in fibromyalgia (FM) by reviewing and compiling findings of nonpharmacological treatment for fatigue in patients with FM. The management of fatigue in FM should incorporate regular physical exercise maintained for a longer period of time, which contributes to improved general health and facilitates coping with fatigue and other symptoms. The evidence is still low and the effect of exercise on fatigue in FM needs to be further studied. Patients with FM appear to benefit from general sleep hygiene advice and also need to develop individual strategies to manage the complex factors in everyday life that may be related to fatigue.

First draft submitted: 11 March 2016; Accepted for publication: 31 May 2016; Published online: 14 June 2016

\section{Background}

- Fatigue in fibromyalgia

The prevalence of fibromyalgia (FM) has been estimated to be $1-3 \%$ of the population in the western world and it is more prevalent in women [1,2]. FM is characterized by widespread pain, tenderness and fatigue; symptoms of psychological distress and cognitive difficulties are also common [2-5]. Women with FM have rated their global fatigue at the same magnitude as pain intensity and fatigue has been found to limit both their social life and their work ability [6-8]. In a recent study, $82 \%$ of nearly 3000 patients with FM reported severe fatigue, which was the highest proportion compared

\section{KEYWORDS}

- exercise • fatigue

- fibromyalgia

- management

- recommendations • review

- sleep • treatment

'University of Gothenburg, Sahlgrenska Academy, Institution of Neuroscience \& Physiology - Section of Health \& Rehabilitation, 
with all the rheumatic diseases included in the study [9]. Fatigue has also been estimated to be the third most distressing symptom in women with FM who are over 50 years of age, following fear of symptoms worsening and difficulty staying asleep [10].

Women with FM have described their fatigue in terms of sleepless nights, social withdrawal, loss of mental energy, physical weakness and overwhelming exhaustion [8]. They have also been shown to report worse symptoms of general, mental and physical aspects of fatigue as well as lack of motivation and reduced activity than have healthy age-matched women [11].

Pain in FM is explained by an amplification of nociceptive input due to central sensitization and impaired central pain inhibition [12]. There is limited neurobiological knowledge about cause of sleep disturbance and fatigue in FM, but they are understood as multifactorial, and partly related to hypersensitivity to unpleasant sensory stimuli [13]. On the other hand, physical deconditioning also contributes to fatigue, especially to a physical dimension of it. Physical activity induces markedly higher fatigue in women with FM than healthy controls [14]. A reason for that might be the fact that women with FM have reduced muscle strength when compared with healthy women [15].

Fatigue in FM is complex and influenced by multiple factors [16]. Different aspects of fatigue have been shown to be associated with sociodemographic aspects such as low age and low working capacity in FM $[1,17]$ as well as other health-related aspects like high pain intensity, increased muscular tenderness, psychological distress, poor sleep quality [11,17-19], a sedentary lifestyle [20] and low level of physical activity and physical function [11,21-22]. Due to this complexity of fatigue, the management and treatment of fatigue is a challenge for the patients with FM as well as the healthcare personnel. This review summarizes findings on nonpharmacological treatments for fatigue in FM and suggests recommendations for the management of fatigue based on the current level of knowledge.

\section{- Assessment of fatigue}

Fatigue is one of the core domains recommended by the OMERACT group to be assessed in all trials with patients with FM but there is no consensus for which tools to use for the assessment $[4,16]$. Although the physiological mechanisms of fatigue are being continuously studied, there are yet no recognized objective measures of fatigue in FM [16]. The subjective experience of fatigue is fluctuating and multidimensional which complicates the assessment of self-reported fatigue [16,23-25]. Fatigue can be assessed as a one-dimensional global concept, especially when screening for fatigue. The visual analog scale (VAS) for fatigue included in the original version of the Fibromyalgia Impact Questionnaire (FIQ) and the revised version of the Fibromyalgia Impact Questionnaire (FIQR) is a commonly used one-dimensional measure [26].

However, the complexity of fatigue favors the use of instruments that include assessment of both the patient's experience of fatigue as well as the effect of fatigue on daily life, especially when the purpose is to evaluate complex interventions $[11,16]$. There is no consensus on how many dimensions of fatigue exist but measures have been recommended to include aspects of physical, emotional and cognitive aspects of fatigue as well as fatigue's impact on work and social activities [16]. Most fatigue scales differ in which and how many dimensions of fatigue they comprise, probably due to the group of patients for which they are constructed.

A numerous of instruments have been used to assess different aspects of fatigue in trials of FM [27,28]. The Multidimensional Fatigue Inventory (MFI-20) is a self-administered instrument that comprises five subscales of fatigue: general fatigue, physical fatigue, mental fatigue, reduced activity and reduced motivation [24]. The MFI-20 was originally constructed for use in patients with cancer but has been validated in Swedish women with FM [29]. The MFI-20 has been used in both exercise studies [30,31] and pharmacological studies in FM [32] and also in studies of several other chronic pain conditions, such as rheumatoid arthritis [33], primary Sjögren's syndrome [34] and ankylosing spondylitis [35]. The Fatigue Severity Scale (FSS) is a one-dimensional measure for self-reported fatigue comprising nine items and the FSS has been recommended for assessment of severity of fatigue in FM due to its robust psychometric properties [36]. Other tools used to assess aspects of fatigue in trials with FM are, for example, the Multidimensional Assessment of Fatigue (MAF) [37] and the ShortForm 36 Vitality subscale [38]. Although some of these instruments have been tested for validity and reliability in FM, they were originally developed for other chronic diseases experiencing 
fatigue. The PROMIS (Patient Reported Outcomes Measurement Information System) has recently developed the PROMIS Fatigue ${ }_{\mathrm{FM}}$ Profile; an FM-specific assessment of multiple aspects of fatigue in FM [39]. The instrument is brief and contains subscales of fatigue experience, fatigue social impact, fatigue motivational impact and fatigue cognitive impact. The PROMIS Fatigue $_{\mathrm{FM}}$ Profile has been shown to possess satisfactory psychometric properties in patients with FM [39].

\section{Treatment of fatigue in FM}

Effects of different types of treatment have been studied thoroughly in FM. Both pharmacological and nonpharmacological treatments have been shown to have an effect on symptom severity and physical function [40-42]. The most efficacious treatment of health problems related to FM requires a multidisciplinary approach combining pharmacological treatment, exercise and cognitive behavioral therapy $[16,40,43]$. The treatments are most successful when tailored individually with consideration to pain, fatigue, function and other features associated with FM $[16,44]$.

Several pharmacological treatments for patients with FM have been found to diminish fatigue in patients with FM, such as anticonvulsants, antidepressants, serotonin reuptake inhibitors and others [45]. Exercise in different forms have also been shown to have positive effects on several symptoms and physical function in patients with FM [46]. In addition, exercise is commonly known to enhance general health, which facilitates coping with pain, fatigue and other common symptoms in FM [47].

FM often co-exists with other diseases associated with fatigue. The prevalence of FM has been reported to be between 11 and $16 \%$ in rheumatic diseases such as rheumatoid arthritis, systemus lupus erythematosus and osteoarthritis, and about $55 \%$ in patients with myalgic encephalomyelitis (ME) [48]. These overlaps make the fatigue in FM even more complex and multifactorial and it is crucial that other disorders known to cause fatigue such as cancer, inflammatory diseases, anemia or hypothyroidism are ruled out and treated before relying on nonpharmacological treatments for fatigue in FM.

\section{- Aerobic exercise}

Aerobic exercise is commonly recommended for patients with FM and is often included in the general guidelines. The early studies were of low-intensity exercise ( $<60 \%$ of $\mathrm{VO}_{2}$ max), often by means of walking, which was adjusted to the patient's limitations and symptoms. Regular exercise often resulted in improvement in symptoms and well-being. Later studies have shown that patients with FM are able to increase the exercise level up to moderate and vigorous intensity, when exercise is initially started at a low level and increase successively. Initial increase of symptom severity often occurs, but it commonly declines after some weeks when exercising at an appropriate frequency and intensity. It is important to discuss the possible increase of symptoms with the patient when planning a new mode of exercise in order to enhance her self-efficacy to manage the exercise program.

Several studies of moderate-intensity to highintensity aerobic exercise by means of cycling, dance or whole-body exercise for patients with fibromyalgia have been published. Aerobic exercise ( $>60 \%$ of $\mathrm{VO}_{2}$ max) performed two- to three-times a week in sessions between 20 and $60 \mathrm{~min}$ for at least 6 weeks has been shown to improve physical capacity in patients with FM. [42,49]. Also aerobic exercise performed at a lower intensity ( $<60 \%$ of $\left.\mathrm{VO}_{2} \max \right)$, has been shown to improve physical capacity in patients with impaired physical function [49].

Aerobic exercise has also been shown to be able to reduce fatigue in patients with FM, however with a small-effect size [49]. For example, a long-term follow-up after supervised 15-week Nordic walking twice a week showed improvements in MFI-20 General and Physical fatigue [31]. Interestingly, same improvement was found in the control group, which had engaged in low-intensive walking. The results indicate that regular exercise over a longer period of time contributes to decreased fatigue in FM, and the regularity may be more crucial for change in fatigue than the intensity of the exercise [31]. There is yet no evidence that aerobic exercise has effect on sleep in FM [49].

\section{- Pool exercise}

Pool exercise is commonly performed in temperate water $\left(30-34^{\circ} \mathrm{C}\right)$ for patients with pain, which appears to reduce pain and stiffness and enhance relaxation. The viscosity of the water provides resistance to the exercises, while the buoyancy facilitates them [50]. Pool exercise is a wide concept and could comprehend both aerobic exercise and exercises aiming to improve strength and flexibility as well as relaxation and meditative 
movements [51]. Temperate pool exercise comprising all, or some, of these components have been suggested to have a positive effect in patients with FM on overall well-being, physical function and different symptoms, such as pain $[30,40,51]$.

While there is yet not sufficient evidence of the effect of pool exercise on fatigue in patients with FM, it has been found to contribute to a moderate improvement on their sleep disturbances [51].

\section{- Resistance exercise}

Resistance exercise has been defined as a type of exercise that requires the body's musculature to move against an opposing force, usually presented by some kind of equipment [52]. Resistance exercise for patients with FM is favorably performed twice a week for at least 15 weeks, starting with loads of $40 \%$ of 1 repetition maximum (RM), with 15-20 repetitions in one to two sets, and increasing the intensity successively until loads reached $80 \%$ of 1 RM, performed with five to eight repetitions in one to two sets [53]. Not all patients with FM manage to exercise with heavy loads, but in a recent exercise study a total of $63 \%$ of the patients with FM reached the exercise level of $80 \%$ of 1 RM [53]. Patients managing loads of $80 \%$ of 1 RM were found to have a better subjective and objective health, which implies that the exercise level has to be adjusted to each patient's resources [53].

Resistance exercise has been found to have a positive effect on muscle strength, self-reported physical function, multidimensional function, pain and fatigue in FM, but not on sleep; however, the evidence of these findings is low [54]. Preliminary results of a randomized controlled study comparing 15 weeks of resistance exercise with relaxation therapy in 130 women with FM indicate that resistance exercise can decrease physical fatigue [55]. In this randomized controlled trial, the resistance exercise program was individually planned together with each participant according to person-centered principals. This implied that the patient's preferences, limitations and obstacles were discussed when determining resistance of equipment in order to improve the sense of control and self-efficacy to manage the program [53,55].

\section{- Meditative movement therapies}

Yoga, tai chi and qigong are ancient movement therapies developed in Asia and aiming for improvement of the quality of movement, awareness, peacefulness, relaxation and the link between body and mind. These meditative movement therapies are increasing in popularity among healthy people. As performance of the movements usually is not strenuous, they are increasing in popularity also among people suffering from pain disorders. Meta-analyses of meditative movement therapies in FM showed improvement in sleep disturbance and fatigue $[56,57]$ Separate analyses of different types of meditative movement therapies indicated that yoga yields best effect on fatigue in FM [49]. Also mindfulness-based stress reduction might be a useful approach for patients with FM [58].

\section{- Multidisciplinary treatment \& patient education}

A multidisciplinary approach has been recommended for management of symptoms in FM $[16,40]$ and multidisciplinary treatment has been shown to improve all core symptoms in FM, including small effects on fatigue and sleep [45].

In FM, education programs are recommended to be multidisciplinary, include information about the nature of fatigue and other symptoms associated with FM and aim to enhance a selfefficacy change in relation to healthy behaviors [16]. Patient education programs should also enhance the patients' skills for managing their symptoms on a day-to-day basis and encourage the patients to practice healthy behaviors that could lead to positive changes in symptoms and health status [59]. Activity pacing is a commonly integrated concept in education programs for patients with FM. Activity pacing for patients with FM involves modifying daily activities to reduce disability due to pain and fatigue and improve general function [60]. A recent meta-analysis have shown that there is yet no evidence that education has an effect on fatigue or sleep in FM [45]. Patient education has been recommended to be combined with cognitive-behavioral techniques and physical exercise to be most effective in FM and [45,59].

\section{- Sleep hygiene}

Low ratings of quality and quantity of sleep are associated with higher levels of multiple dimension of fatigue in FM [11]. Instructions of sleep hygiene for patients with FM have been found to contribute to improvement in pain intensity, fatigue and subjective sleep quality [61]. Sleep hygiene instructions include advice to have 
regular sleeping routines; avoid coffee, tea, food, alcohol, smoking and watching TV close to bedtime; regulate the sleeping environment such as having a comfortable bed and optimal room temperature; avoid light and loud noise and be regularly physically active, however, not to close to bedtime [61].

\section{Discussion}

\section{- Physical exercise}

Regular physical exercise contributes to improved general health which facilitates coping with pain, fatigue and other symptoms. Physical exercise in forms of aerobic exercise, pool exercise, resistance exercise and meditative movement therapies has shown promising effects on fatigue and/or sleep disturbances, especially when the exercise is maintained for a longer period of time. However, the evidence is still low and need to be further studied.

It is crucial to inform the patients about potential obstacles for maintenance of exercise, such as risk of initially increased pain and that improvements may take longer time to achieve than for persons not having FM.

\section{- Person-centered approach}

Due to the variety of symptom severity in patients with FM, the exercise needs to be adjusted to each patient's resources and limitations. Furthermore, as fatigue is a complex and fluctuating concept and is influenced by individual factors, a successful treatment has to be planned together with the patient. An example of this type of approach is person-centered care [62] and person-centered physiotherapy. Person-centeredness implies that the patient is assumed to be an expert of her/his body and specific health problems, while the therapist possesses professional knowledge about the disorder and treatment options. Person-centered approach in physiotherapy is started with sharing knowledge and information, during which the patient is invited to describe her/his health problems and her life situation, including factors influencing symptoms, such as fatigue [63]. Patient's earlier experiences of physical activity, potential hinders for activity as well as preferences and goals give valuable basis for shared decision on how to plan exercise. Examinations and exercise instructions, adjustments and modifications appear to work well when based on self-efficacy principals, in which exercise is started at low intensity and light loads [53]. When the program has been tried out, together with the patient, a structured health plan, including dose, duration and intensity of exercise is written down. This is done together with the patient to suit her circumstances. The goals of exercise and other practices are included in a written health plan/exercise plan, and subsequent meetings are planned in to evaluate and modify it [63].

\section{- Multidisciplinary patient education}

There is yet no evidence that patient education programs contribute to specific improvement of fatigue in FM, according to a recent metaanalysis [45]. However, the content of the education programs vary in the studies included in the meta-analysis, which makes it even harder to draw conclusions of the effect of education.

Multidisciplinary patient education aiming to increase knowledge about symptoms in FM, enhance self-efficacy and stimulate exercise and healthy behaviors could contribute to improved general health. It is likely that improved general health over time facilitates coping with fatigue as well as other symptoms associated with FM.

\section{- Sleep hygiene}

Patients with FM appear to benefit from general sleep hygiene advice [61], which often is forgotten by healthcare providers. The advice incorporates establishing regular sleep habits and optimizing sleep environments as well as minimizing disturbances and stressors prior to bedtime [61]. A dialogue between the therapist and the patient about sleep habits and sleep environment should preferably be included in the treatment of fatigue in FM.

\section{Future perspective}

Nonpharmacological treatments and practices for management of fatigue on FM and related disorders is a new field in the healthcare. Several intervention studies show promising results, but there is lack of knowledge about effects of treatment and management strategies for specific patient groups and subgroups with specific characteristics. More research is needed about minimal amount of exercise/practice required to receive a beneficial effect of exercise, meditative movement or other practices.

There is also lack of knowledge about physiological response to nonpharmacological treatment options. Research from a physiological and a clinical perspective should be combined to develop new knowledge. 


\section{Conclusion}

The management of fatigue in FM should incorporate regular physical exercise, practicing general sleep hygiene and individual strategies to manage those complex factors in everyday life that may be related to fatigue. As fatigue interacts with several other symptoms in FM, such as pain, depression and anxiety, the recommendations in this review are likely to enhance management of other common symptoms in FM as well.

Financial \& competing interests disclosure

Financial support for the study was received from the Medical Faculty of Göteborg University (ALF) and the
University of Gothenburg Centre for Person Centered Care (GPCC). The authors have no other relevant affiliations or financial involvement with any organization or entity with a financial interest in or financial conflict with the subject matter or materials discussed in the manuscript apart from those disclosed.

No writing assistance was utilized in the production of this manuscript.

\section{Open access}

This work is licensed under the Creative Commons Attribution-NonCommercial 3.0 Unported License. To view a copy of this license, visit http://creativecommons.org/ licenses/by-nc-nd/3.0/

\section{References}

Papers of special note have been highlighted as:

- of interest; $\bullet \bullet$ of considerable interest

1 Gran JT. The epidemiology of chronic generalized musculoskeletal pain. Best Pract. Res. Clin. Rheumatol. 17(4), 547-561 (2003).

2 Wolfe F, Ross K, Anderson J, Russell IJ, Hebert $\mathrm{L}$. The prevalence and characteristics of fibromyalgia in the general population. Arthritis Rheum. 38(1), 19-28 (1995).

3 Wolfe F, Smythe HA, Yunus MB et al. The American College of Rheumatology 1990 Criteria for the Classification of Fibromyalgia. Report of the Multicenter Criteria Committee. Arthritis Rheum. 33(2), 160-172 (1990).

4 Mease P, Arnold LM, Choy EH et al. Fibromyalgia syndrome module at OMERACT 9: domain construct. J. Rheumatol. 36(10), 2318-2329 (2009).

5 Rohrbeck J, Jordan K, Croft P. The frequency and characteristics of chronic widespread pain in general practice: a case-control study. Br. J. Gen. Pract. 57(535), 109-115 (2007).

6 Wuytack F, Miller P. The lived experience of fibromyalgia in female patients, a phenomenological study. Chiropr. Man. Therap. 19(1), 22 (2011).

7 Liedberg GM, Henriksson CM. Factors of importance for work disability in women with fibromyalgia: an interview study. Arthritis Rheum. 47(3), 266-274 (2002).

8 Sallinen M, Kukkurainen ML, Peltokallio L, Mikkelsson M. "I'm tired of being tired" fatigue as experienced by women with fibromyalgia. Adv. Physiother. 13, 11-17 (2011).

9 Overman CL, Kool MB, Da Silva JA, Geenen $R$. The prevalence of severe fatigue in rheumatic diseases: an international study. Clin. Rheumatol. doi:10.1007/s10067-0153035-6 (2015) (Epub ahead of print).

10 Shillam CR, Dupree Jones K, Miller L. Fibromyalgia symptoms, physical function, and comorbidity in middle-aged and older adults. Nurs. Res. 60(5), 309-317 (2011).

11 Ericsson A, Bremell T, Mannerkorpi K Usefulness of multiple dimensions of fatigue in fibromyalgia. J. Rehabil. Med. 45(7), 685-693 (2013).

12 Lannersten L, Kosek E. Dysfunction of endogenous pain inhibition during exercise with painful muscles in patients with shoulder myalgia and fibromyalgia. Pain 151(1), 77-86 (2010).

13 Ceko M, Bushnell MC, Gracely RH. Neurobiology underlying fibromyalgia symptoms. Pain Res. Treat. 2012, 585419 (2012).

14 Christidis N, Ghafouri B, Larsson A et al. Comparison of the levels of proinflammatory cytokines released in the vastus lateralis muscle of patients with fibromyalgia and healthy controls during contractions of the quadriceps muscle - a microdialysis study. PLoS ONE 10(12), e0143856 (2015).

15 Maquet D, Croisier JL, Renard C, Crielaard JM. Muscle performance in patients with fibromyalgia. Joint Bone Spine 69 (3), 293-299 (2002).

16 Vincent A, Benzo RP, Whipple MO, McAllister SJ, Erwin PJ, Saligan LN. Beyond pain in fibromyalgia: insights into the symptom of fatigue. Arthritis Res. Ther. 15(6), 221 (2013).

- Comprehensive review about assessment and treatment of fatigue in fibromyalgia (FM).

17 Wolfe F, Hawley DJ, Wilson K. The prevalence and meaning of fatigue in rheumatic disease. J. Rheumatol. 23(8), 1407-1417 (1996).

18 Kurtze N, Svebak S. Fatigue and patterns of pain in fibromyalgia: correlations with anxiety, depression and co-morbidity in a female county sample. Br. J. Med. Psychol. 74(Pt 4), 523-537 (2001).

19 Nicassio PM, Moxham EG, Schuman CE, Gevirtz RN. The contribution of pain, reported sleep quality, and depressive symptoms to fatigue in fibromyalgia. Pain 100 (3), 271-279 (2002).

20 Segura-Jimenez V, Borges-Cosic M, SorianoMaldonado A et al. Association of sedentary time and physical activity with pain, fatigue, and impact of fibromyalgia: the al-Andalus study. Scand. J. Med. Sci. Sports doi:10.1111/ sms.12630 (2015) (Epub ahead of print).

21 Rutledge DN, Jones K, Jones CJ. Predicting high physical function in people with fibromyalgia. J. Nurs. Scholarsh. 39(4), 319-324 (2007).

22 Kop WJ, Lyden A, Berlin AA et al. Ambulatory monitoring of physical activity and symptoms in fibromyalgia and chronic fatigue syndrome. Arthritis Rheum. 52(1), 296-303 (2005).

23 Guymer EK, Clauw DJ. Treatment of fatigue in fibromyalgia. Rheum. Dis. Clin. North Am. 28(2), 367-378 (2002).

24 Smets EM, Garssen B, Bonke B, De Haes JC. The multidimensional fatigue inventory (MFI) psychometric qualities of an instrument to assess fatigue. J. Psychosom. Res. 39(3), 315-325 (1995).

25 Watt T, Groenvold M, Bjorner JB, Noerholm $\mathrm{V}$, Rasmussen NA, Bech P. Fatigue in the Danish general population. Influence of sociodemographic factors and disease. J. Epidemiol. Community Health 54(11), 827-833 (2000). 
26 Bennett RM, Friend R, Jones KD, Ward R, Han BK, Ross RL. The revised fibromyalgia impact questionnaire (FIQR): validation and psychometric properties. Arthritis Res. Ther. 11(4), R120 (2009).

27 Choy EH, Arnold LM, Clauw DJ et al. Content and criterion validity of the preliminary core dataset for clinical trials in fibromyalgia syndrome. J. Rheumatol. 36(10), 2330-2334 (2009).

28 Vincent A, Hoskin TL, Whipple MO et al. OMERACT-based fibromyalgia symptom subgroups: an exploratory cluster analysis. Arthritis Res. Ther. 16(5), 463 (2014).

29 Ericsson A, Mannerkorpi K. Assessment of fatigue in patients with fibromyalgia and chronic widespread pain. Reliability and validity of the Swedish version of the MFI-20. Disabil. Rehabil. 29(22), 1665-1670 (2007).

30 Mannerkorpi K, Nordeman L, Ericsson A, Arndorw M. Pool exercise for patients with fibromyalgia or chronic widespread pain: a randomized controlled trial and subgroup analyses. J. Rehabil. Med. 41(9), 751-760 (2009).

31 Mannerkorpi K, Nordeman L, Cider A, Jonsson G. Does moderate-to-high intensity Nordic walking improve functional capacity and pain in fibromyalgia? A prospective randomized controlled trial. Arthritis Res. Ther. 12(5), R189 (2010).

32 Arnold LM, Clauw D, Wang F, Ahl J, Gaynor PJ, Wohlreich MM. Flexible dosed duloxetine in the treatment of fibromyalgia: a randomized, double-blind, placebo-controlled trial. J. Rheumatol. 37(12), 2578-2586 (2010).

33 Rupp I, Boshuizen HC, Jacobi CE, Dinant HJ, Van Den Bos GA. Impact of fatigue on health-related quality of life in rheumatoid arthritis. Arthritis Rheum. 51(4), 578-585 (2004).

34 Barendregt PJ, Visser MR, Smets EM et al. Fatigue in primary Sjogren's syndrome. Ann. Rheum. Dis. 57(5), 291-295 (1998).

35 Van Tubergen A, Coenen J, Landewe R et al. Assessment of fatigue in patients with ankylosing spondylitis: a psychometric analysis. Arthritis Rheum. 47(1), 8-16 (2002).

36 Boomershine CS. A comprehensive evaluation of standardized assessment tools in the diagnosis of fibromyalgia and in the assessment of fibromyalgia severity. Pain Res. Treat. 2012, 653714 (2012).

37 Belza BL. Comparison of self-reported fatigue in rheumatoid arthritis and controls. J. Rheumatol. 22(4), 639-643 (1995).
38 Ware JE Jr., Sherbourne CD. The MOS 36-item short-form health survey (SF-36). I. Conceptual framework and item selection. Med. Care 30 (6), 473-483 (1992).

39 Kratz AL, Schilling S, Goesling J, Williams DA. The PROMIS Fatigue Profile: a self-report measure of fatigue for use in fibromyalgia. Qual. Life Res. doi:10.1007/s11136-016-1230-9 (2016) (Epub ahead of print).

40 Carville SF, Arendt-Nielsen S, Bliddal H et al. EULAR evidence-based recommendations for the management of fibromyalgia syndrome. Ann. Rheum. Dis. 67(4), 536-541 (2008).

- Important clinical recommendations for patients with FM.

41 Mannerkorpi K, Henriksson C. Nonpharmacological treatment of chronic widespread musculoskeletal pain. Best Pract. Res. Clin. Rheumatol. 21(3), 513-534 (2007).

42 Busch AJ, Barber KA, Overend TJ, Peloso PM, Schachter CL. Exercise for treating fibromyalgia syndrome. Cochrane Database Syst. Rev. (3), CD003786 (2007).

- Comprehensive review of exercise for patients with FM.

43 Sarzi-Puttini P, Atzeni F, Salaffi F, Cazzola M, Benucci M, Mease PJ. Multidisciplinary approach to fibromyalgia: what is the teaching? Best Pract. Res. Clin. Rheumatol. 25(2), 311-319 (2011).

44 Busch AJ, Webber SC, Brachaniec M et al. Exercise therapy for fibromyalgia. Curr. Pain Headache Rep. 15(5), 358-367 (2011).

45 Papadopoulou D, Fassoulaki A, Tsoulas C, Siafaka I, Vadalouca A. A meta-analysis to determine the effect of pharmacological and nonpharmacological treatments on fibromyalgia symptoms comprising OMERACT-10 response criteria. Clin. Rheumatol. 35(3), 573-586 (2015).

- Comprehensive meta-analysis of treatments for patients with FM.

46 Bidonde J, Busch AJ, Bath B, Milosavljevic S. Exercise for adults with fibromyalgia: an umbrella systematic review with synthesis of best evidence. Curr. Rheumatol. Rev. 10(1), 45-79 (2014).

47 Mannerkorpi K, Gard G. Physiotherapy group treatment for patients with fibromyalgia-an embodied learning process. Disabil. Rehabil. 25(24), 1372-1380 (2003).

48 Yunus MB. The prevalence of fibromyalgia in other chronic pain conditions. Pain Res. Treat. 2012584573 (2012).
49 Hauser W, Klose P, Langhorst J et al. Efficacy of different types of aerobic exercise in fibromyalgia syndrome: a systematic review and meta-analysis of randomised controlled trials. Arthritis Res. Ther. 12(3), R79 (2010).

- Comprehensive review of exercise for patients with FM.

50 Mannerkorpi K, Iversen MD. Physical exercise in fibromyalgia and related syndromes. Best Pract. Res. Clin. Rheumatol. 17(4), 629-647 (2003).

51 Bidonde J, Busch AJ, Webber SC et al. Aquatic exercise training for fibromyalgia. Cochrane Database Syst. Rev. 10, CD011336 (2014).

52 Fleck SJ, Kraemer JW. Designing Resistance Training Programs (3rd Edition). Human Kinetics, Leeds, UK (2004).

53 Larsson A, Palstam A, Lofgren M et al. Resistance exercise improves muscle strength, health status and pain intensity in fibromyalgia-a randomized controlled trial. Arthritis Res. Ther. 17, 161 (2015).

54 Busch AJ, Webber SC, Richards RS et al. Resistance exercise training for fibromyalgia. Cochrane Database Syst. Rev. 12, CD010884 (2013).

55 Ericsson A, Palstam A, Larsson A et al. Resistance exercise improves multidimensional fatigue in women with fibromyalgia. A randomized controlled trial. Ann. Rheum. Dis. 74(Suppl. 2), 180-181 (2015).

56 Langhorst J, Klose P, Dobos GJ, Bernardy K, Hauser W. Efficacy and safety of meditative movement therapies in fibromyalgia syndrome: a systematic review and metaanalysis of randomized controlled trials. Rheumatol. Int. 33(1), 193-207 (2013).

57 Mist SD, Firestone KA, Jones KD. Complementary and alternative exercise for fibromyalgia: a meta-analysis. J. Pain Res. 6, 247-260 (2013).

58 Lauche R, Cramer H, Dobos G, Langhorst J, Schmidt $S$. A systematic review and meta-analysis of mindfulness-based stress reduction for the fibromyalgia syndrome. J. Psychosom. Res. 75(6), 500-510 (2013).

59 Burckhardt CS. Educating patients: self-management approaches. Disabil. Rehabil. 27(12), 703-709 (2005).

60 Antcliff D, Keeley P, Campbell M, Woby S, McGowan L. Exploring patients' opinions of activity pacing and a new activity pacing questionnaire for chronic pain and/or fatigue: a qualitative study. Physiotherapy doi:10.1016/j. physio.2015.08.001 (2015) (Epub ahead of print). 
61 Orlandi AC, Ventura C, Gallinaro AL, Costa RA, Lage LV. Improvement in pain, fatigue, and subjective sleep quality through sleep hygiene tips in patients with fibromyalgia. Rev. Bras. Reumatol. 52(5), 666-678 (2012).
62 Ekman I, Swedberg K, Taft C et al. Personcentered care - ready for prime time. Eur. J. Cardiovasc. Nurs. 10(4), 248-251 (2011).

63 Feldthusen C, Dean E, Forsblad-D'elia H, Mannerkorpi K. Effects of person-centered physical therapy on fatigue-related variables in persons with rheumatoid arthritis: a randomized controlled trial. Arch Phys. Med. Rehabil. 97(1), 26-36 (2016). 